\title{
Role of the endothelium in modulating functional responses of isolated bovine anterior ciliary arteries to vasoconstrictor agonists
}

\author{
Christine H Buckley, Patrick W F Hadoke, Colm J O’Brien
}

\begin{abstract}
Backgroundlaims-Endothelium dependent vasodilatation is an important regulator of blood flow to the eye but its role has not been investigated in vessels supplying the ciliary body. This study assessed the role of the endothelium in modulating vasoconstrictor responses of the intraocular bovine anterior ciliary artery.

Methods-Bovine anterior ciliary arteries $(n=33)$ were mounted in a myograph, containing physiological salt solution at $37^{\circ} \mathrm{C}$, for isometric force measurement. Cumulative concentration-response curves were obtained to the constrictor agonists 5-hydroxytryptamine (5-HT), noradrenaline, phenylephrine, prostaglandin $F_{2 \alpha}$, endothelin-1, and $\mathrm{KCl}$ in both endothelium intact and denuded arteries.

Results-All vasoconstrictors produced sustained contractile responses which were unaffected by the removal of the endothelium. Responses to 5-HT were also unaffected by inhibition of nitric oxide synthase.

Conclusion-These results indicate that neither agonist stimulated nor basal release of nitric oxide from the endothelium modulates responses to vasoconstrictor agonists in the isolated bovine anterior ciliary artery when measured in a no flow isometric system.

(Br F Ophthalmol 1998;82:826-829)
\end{abstract}

Basal and/or agonist induced release of endothelium derived nitric oxide (NO) is a significant factor in the regulation of ocular blood flow. ${ }^{1-3}$ Consequently endothelial cell dysfunction in ocular vessels may account for the ophthalmic complications of diseases associated with dysfunction of the endothelium in the systemic vasculature (for example, diabetes, hypertension). ${ }^{4}$ Similar alterations may also occur in some patients with glaucoma who have ocular or generalised vasospastic sequelae. $^{5}$

The role of endothelium derived mediators in the control of vascular function in the eye varies in different arteries. ${ }^{2}$ Their contribution to tone in vessels supplying the ciliary body has not been investigated, although high levels of constitutive NO synthase (eNOS) are located in the human outflow pathway and ciliary muscle. ${ }^{6}$ This may be relevant to the development of glaucoma as patients with this condition, which is associated with an increase in both outflow resistance and intraocular pressure (IOP), have a reduced distribution of NO containing cells in the outflow pathway. ${ }^{7}$ Furthermore, nitrovasodilators, which mimic the actions of NO, reduce IOP through a mechanism involving alterations in the resistance to aqueous humour outflow. ${ }^{8}$

The limited data available on normal physiological control of ocular blood vessels make it difficult to describe the exact vascular alterations which occur during disease progression. The development of the small vessel myograph by Mulvany and Halpern ${ }^{9}$ has provided a technique which can be used to investigate vascular function in vitro using vessels with an internal diameter of as little as $100 \mu \mathrm{m}$. The aim of the present study was to investigate the role of the endothelium in mediating vasoconstriction in the intraocular bovine anterior ciliary artery.

\section{Materials and methods}

TISSUE PREPARATION

Bovine eyes were obtained from the abattoir and transported in cold physiological salt solution (PSS) of the following composition ( $\mathrm{mM}$ ): $\mathrm{NaCl} 119, \mathrm{KCl} 4.7, \mathrm{MgSO}_{4} 1.17, \mathrm{KH}_{2} \mathrm{PO}_{4}$ 1.18, glucose 5.5, $\mathrm{K}_{2}$ EDTA $0.026, \mathrm{NaHCO}_{3}$ $25, \mathrm{CaCl}_{2}$ 2.5. The posterior segment of the eye together with the vitreous was removed, intraocular anterior ciliary arteries were dissected from beneath the choroid and transferred to the chamber of a wire myograph (Model 400A, JP Trading, Aarhus, Denmark).

Arterial rings, approximately $2 \mathrm{~mm}$ in length, were mounted on two $40 \mu \mathrm{m}$ intraluminal wires for measurement of isometric force development. The vessels were allowed to equilibrate in PSS at $37^{\circ} \mathrm{C}$ and gassed with $95 \%$ oxygen, $5 \%$ carbon dioxide for 30 minutes before undergoing normalisation using standard methodology. ${ }^{10}$ Briefly, this involved stepwise stretching of the vessel and application of the LaPlace relation to determine the internal circumference $\left(\mathrm{L}_{100}\right)$ of a vessel when relaxed and under an effective transmural pressure of $100 \mathrm{~mm} \mathrm{Hg}(13.3 \mathrm{kPa})$. The vessel was then set at an internal circumference of $0.9 \mathrm{~L}_{100}$ at which many small arteries, ${ }^{10}$ including bovine ${ }^{11}$ and canine ${ }^{12}$ ocular arteries develop maximum or near maximum active tension. This setting has been used previously in functional investigations of bovine anterior ciliary arteries. ${ }^{13}$ The vessels were then left to equilibrate under their normalised tension for 30 minutes.

The functional integrity of the endothelium was assessed in each artery by the addition of bradykinin $\left(\mathrm{BK} ; 10^{-6} \mathrm{M}\right)$ following contraction 
with 5-hydroxytryptamine (5-HT; $\left.3 \times 10^{-7} \mathrm{M}\right)$. The endothelium was considered intact if $\mathrm{BK}$ evoked a relaxation of greater than $60 \%$ of the 5-HT induced tone. In some arteries the endothelium was removed by gently rubbing the lumen of the vessel with a human hair. Histological studies have confirmed that this method successfully removes the endothelium ${ }^{14}$ and denudation was confirmed in the present study by the failure of the vessel to relax in response to $\mathrm{BK}\left(10^{-6} \mathrm{M}\right)$.

INFLUENCE OF THE ENDOTHELIUM ON VASOCONSTRICTOR RESPONSES

The role played by the endothelium in modulating functional responses of bovine anterior ciliary arteries to vasoconstrictors was investigated using a variety of agonists. Cumulative concentration-response curves were obtained to the vasoconstrictors 5-HT $\left(10^{-9}-3 \times\right.$ $\left.10^{-5} \mathrm{M}\right)$, noradrenaline (NA; $10^{-9}-3 \times 10^{-5} \mathrm{M}$ ), phenylephrine (PE; $\left.10^{-8}-3 \times 10^{-3} \mathrm{M}\right)$, prostaglandin $(\mathrm{PG}) \quad \mathrm{F}_{2 \alpha}\left(10^{-8}-3 \times 10^{-5} \mathrm{M}\right)$, endothelin-1 (ET-1; 10 10 $\left.-3 \times 10^{-7} \mathrm{M}\right)$, and $\mathrm{KCl}(10-125 \mathrm{mM})$ in both endothelium intact and denuded vessels. Following each concentration-response curve the vessels were washed thoroughly with PSS and allowed to relax fully for at least 20 minutes before the next drug was tested.

The role of endothelium derived $\mathrm{NO}$ in the modulation of 5-HT induced contraction was assessed by constructing concentrationresponse curves in the absence of, and following incubation of arteries for 45 minutes with, the $\mathrm{NO}$ synthase inhibitor $\mathrm{N}^{\mathrm{G}}$-nitro-L-arginine $\left(\mathrm{L}-\mathrm{NNA} ; 10^{-4} \mathrm{M}\right)$.

STATISTICAL ANALYSIS

Contractile responses of the vessels were expressed as active wall tension $(\mathrm{mN} / \mathrm{mm})$ and relaxation responses as a percentage of the induced contraction. For each concentrationresponse curve the molar concentration required to produce $50 \%$ of the maximum contraction $\left(\mathrm{EC}_{50}\right)$ was calculated by fitting the data to the Hill equation using the curve fitting program FIG P (Biosoft, UK). The sensitivity of the vessels to each constrictor agonist was expressed as the negative $\log \mathrm{EC}_{50}\left(\mathrm{pD}_{2}\right)$. The results are given as the mean (SEM) for $n$ separate experiments; only one artery was used from each animal and so $\mathrm{n}$ also refers to the number of animals used.

Maximum contraction and $\mathrm{EC}_{50}$ values were compared using Student's unpaired $t$ test and considered significantly different when $\mathrm{p} \leqslant 0.05$.

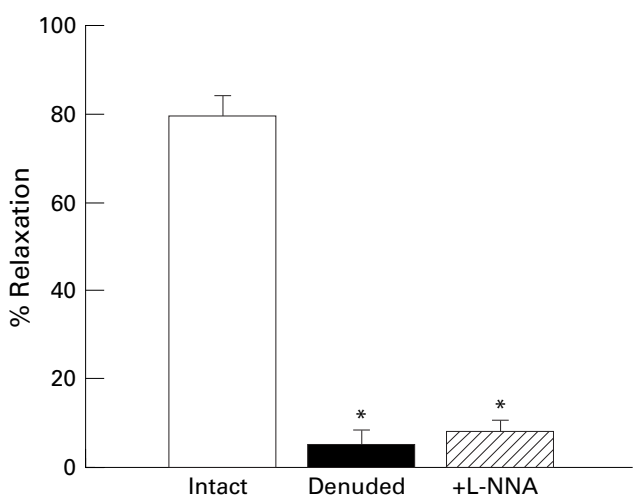

Figure 1 Responses to bradykinin $\left(10^{-6} \mathrm{M}\right)$ in endothelium intact bovine anterior ciliary arteries precontracted with $5-H T\left(3 \times 10^{-7} \mathrm{M}\right)$ in the absence (open column; $n=11$ ) and presence (hatched column; $n=8$ ) of L-NNA $\left(10^{-4} \mathrm{M}\right)$, and in endothelium denuded vessels (solid column; $n=8$ ). Each column represents a mean value with vertical bars indicating SEM. ${ }^{*} p<0.01$ compared with endothelium intact vessels without $L-N N A$.

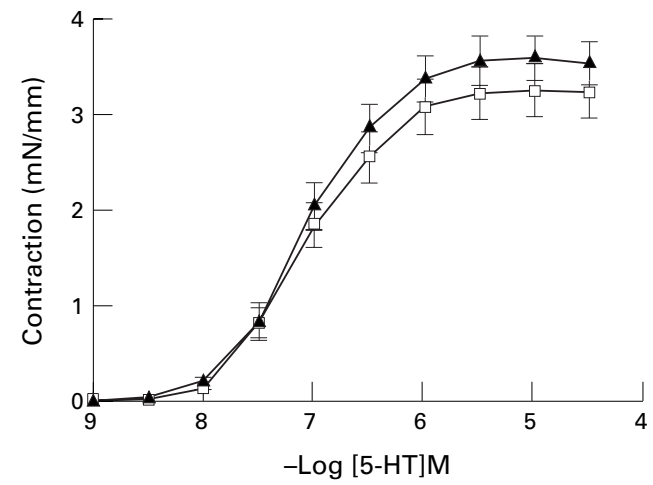

Figure 2 Cumulative concentration-response curves to 5-HT in endothelium intact $(\mathbf{\Delta} ; n=12)$ and denuded ( $\square$; $n=10)$ bovine anterior ciliary arteries. All values are means (SEM).

\section{Results}

Preliminary experiments demonstrated that vessels remained viable if stored in PSS at $4^{\circ} \mathrm{C}$ for 24 hours, with both vasoconstrictor and vasodilator function unaltered (data not shown). This extended the period during which experiments could be performed. The mean normalised lumen diameter of vessels used in the present study was 218 (6) $\mu \mathrm{m}$ $(n=33)$. Vessels which had not had the endothelium removed relaxed by $79.8 \%$ $(4.5 \%)(n=11)$ in response to $\mathrm{BK}$, whereas in denuded arteries the relaxation was reduced to $5.1 \%(3.3 \%)(n=8)($ Fig 1$)$.

In quiescent arteries with an intact endothelium all six agonists tested, 5-HT, NA, PE, $\mathrm{PGF}_{2 \alpha}, \mathrm{ET}-1$, and $\mathrm{KCl}$, produced concentra-

Table 1 Effect of endothelium removal on constrictor responses of bovine anterior ciliary arteries

\begin{tabular}{|c|c|c|c|c|c|c|}
\hline & \multicolumn{3}{|c|}{ Maximum contraction $\left(E_{\text {max }}\right)(\mathrm{mN} / \mathrm{mm})$} & \multicolumn{3}{|l|}{$p D_{2}$} \\
\hline & Intact & Denuded & $p$ Value & Intact & Denuded & $p$ Value \\
\hline 5-HT & $3.65(0.25)(12)$ & $3.26(0.27)(10)$ & 0.30 & $7.04(0.08(12))$ & $7.07(0.07)(10)$ & 0.84 \\
\hline NA & $2.78(0.28)(9)$ & $2.64(0.33)(9)$ & 0.75 & $6.70(0.05)(9)$ & $6.62(0.18)(9)$ & 0.54 \\
\hline $\mathrm{PE}$ & $3.18(0.35)(8)$ & $2.45(0.20)(7)$ & 0.10 & $4.64(0.19)(8)$ & $4.54(0.20)(7)$ & 0.73 \\
\hline $\mathrm{PGF}_{2 u}$ & $2.83(0.31)(8)$ & $2.57(0.44)(5)$ & 0.63 & $5.28(0.12)(8)$ & $5.36(0.22)(5)$ & 0.73 \\
\hline ET-1 1 & $4.21(0.24)(9)$ & $3.67(0.48)(10)$ & 0.34 & $8.17(0.06)(9)$ & $8.26(0.06)(10)$ & 0.36 \\
\hline $\mathrm{KCl}$ & $4.08(0.31)(8)$ & $3.51(0.39)(5)$ & 0.28 & $1.55(0.03)(8)$ & $1.56(0.05)(5)$ & 0.84 \\
\hline
\end{tabular}

Values are expressed as mean (SEM) (n). p Values comparing maximum contractions and $\mathrm{pD}_{2}$ values in endothelium intact versus denuded arteries. 


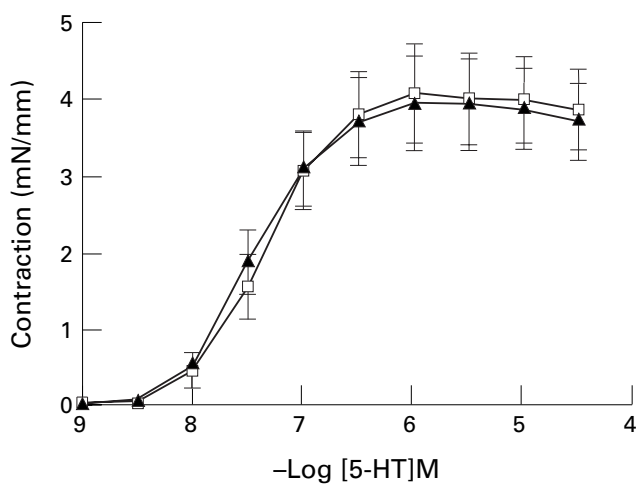

Figure 3 Cumulative concentration-response curves to 5-HT in endothelium intact bovine anterior ciliary arteries incubated for 45 minutes with the nitric oxide synthase inhibitor L-NNA $\left(10^{-4} M ; \mathbf{\Delta} ; n=4\right)$ and in vehicle treated controls $(\square ; n=4)$. All values are means (SEM).

tion dependent contractions (Table 1). Concentration-response curves to 5-HT in both endothelium intact and denuded arteries are shown in Figure 2. A similar pattern of response was seen for each of the other five agonists, except for PE and $\mathrm{PGF}_{2 \alpha}$, which failed to reach a stable maximum contraction in the concentration ranges used. Consequently, the $\mathrm{pD}_{2}$ values calculated for $\mathrm{PE}$ and $\mathrm{PGF}_{2 \alpha}$ (Table 1) are only approximate. Removal of the endothelium did not alter the sensitivity $\left(\mathrm{pD}_{2}\right)$ to any of the agonists (Table 1 ). In all cases, the maximum contractile response was slightly, but not significantly, reduced in endothelium denuded arteries compared with intact vessels (Table 1).

Addition of $10^{-4} \mathrm{M}$ L-NNA (a concentration shown to inhibit BK mediated relaxation in the anterior ciliary artery (Fig 1)) did not cause an increase of tone in any of the isolated arteries (data not shown). Furthermore, incubation with L-NNA $\left(10^{-4} \mathrm{M}\right)$ did not alter the sensitivity or the magnitude of the contraction induced by 5 -HT (Fig 3).

\section{Discussion}

This investigation used small vessel myography to investigate the role of the endothelium in modulating agonist induced vasoconstriction in the bovine anterior ciliary artery. The results have demonstrated that this artery contracts in response to a variety of agonists and indicate that neither basal nor agonist induced release of NO from the endothelium modulates this contraction.

The vascular endothelium makes a major contribution to local vascular tone and blood flow through the release of both vasodilator (NO and prostacyclin) and vasoconstrictor (ET-1 and cyclo-oxygenase products) agents. ${ }^{15}$ Any disturbance to the balance between constrictor and dilator compounds released by the endothelium may result in vasospasm and alterations in blood flow. ${ }^{4}$ Furthermore, many agonists which contract healthy arteries may also contribute to vasodilatation by the release of NO from the endothelium. In arteries with dysfunctional endothelium the vasodilator responses are lost, exacerbating the vasoconstrictor response. ${ }^{16}$ Therefore, endothelial cell dys- function may alter both the basal vascular tone and the response of the vessel to circulating agonists and pharmacological agents and, consequently, is an important factor in both the development and treatment of disorders of the eye.

Functional responses of ocular vessels are heterogeneous, depending upon the anatomical origin of the vessel, its location within or outside the orbit, and also upon the part of the vessel selected (reviewed by Buckley et $a l^{17}$ ). In the present investigation, vasoconstrictor agonists were used to provide information on the role of the endothelium in modulating the action of agonists derived from sympathetic nerves, aggregating platelets and damaged endothelial cells. ${ }^{18} \mathrm{KCl}$, a receptor independent vasoconstrictor, was included as a control and $\mathrm{PGF}_{2 \alpha}$ was also included as many investigations have used this to precontract vessels for subsequent investigation of vasodilator activity. ${ }^{13} 19$ These agonists all produced sustained, concentration dependent responses that were not altered significantly by removal of the endothelium. The slight, but not significant, reduction in the maximum contraction following removal of the endothelium was probably due to slight damage to the vascular smooth muscle cells which is a recognised problem encountered when denuding small arteries. This was supported by the demonstration that inhibition of NO synthase using L-NNA did not result in a reduction of the magnitude of 5-HT induced contraction. Further experiments are required to investigate the effect of NO synthase inhibition on the responses to other constrictor agonists and also to determine the potential role of other vasoactive mediators such as $\mathrm{PGI}_{2}$ in regulating blood flow in this artery. These results suggest that basal release of NO, or any other vasoactive agent, is not a significant factor in bovine anterior ciliary arteries in an isometric system. This observation is supported by the absence of an increase in basal tone following the addition of the L-arginine analogue (L-NNA). In contrast, in vivo experiments using NO synthase inhibitors have demonstrated a significant reduction in blood flow to the anterior uvea in both rabbits and dogs indicating that basal NO release is involved in the regulation of uveal blood flow under resting conditions. ${ }^{2021}$ Whether this difference reflects the different species used or the size of the vessels or is due to the different methodological approaches used requires further investigation. Investigations using porcine isolated ocular arteries have suggested that the influence of the endothelium and endothelium derived NO on contractile responses is dependent upon the size and location of a particular vessel. ${ }^{12}$ Basal release of NO is more important in larger arteries, modulating vasoconstriction in porcine ophthalmic, but not ciliary, arteries. ${ }^{2}$ The results reported for the porcine ciliary artery ${ }^{2}$ are consistent with those obtained in the present study, whether the influence of the endothelium or endothelium derived NO on contractile response depends on vessel size in the bovine ophthalmic 
vascular bed, or more importantly in the human, remains to be determined.

The use of NA and PE demonstrated that $\alpha$ adrenoceptors were present in the anterior ciliary artery. Sensitivity to the specific $\alpha_{1}$ adrenoceptor agonist, PE, was 100-fold lower than that to NA, suggesting that the latter may contract this vessel by stimulation of more than one receptor subtype. Indeed, bovine intraocular long posterior ciliary arteries have been shown to contract in response to both selective $\alpha_{1}$ and $\alpha_{2}$ adrenoceptor agonists. ${ }^{13}$ In contrast, $\alpha_{2}$ adrenoceptor agonists do not cause contraction in canine or human ciliary arteries with the contraction to NA in these vessels mediated solely by $\alpha_{1}$ adrenoceptors. ${ }^{22-25}$ NA can also relax some arteries by stimulating $\beta$ adrenoceptors or by $\alpha_{2}$ adrenoceptor mediated release of NO from the endothelium. ${ }^{26}$ As NA mediated vasoconstriction was unaffected by removal of the endothelium in the present study, $\alpha_{2}$ adrenoceptor mediated release of NO appears not to play a role in regulating tone in the bovine anterior ciliary artery.

The inhibitory action of $\mathrm{NO}$ on platelet aggregation may become impaired in patients with endothelial cell dysfunction, allowing aggregating platelets to adhere to the vascular wall (releasing vasoactive agonists, including $5-\mathrm{HT}$ ), with consequent vasospasm. ${ }^{27}$ The 5-HT induced vasoconstriction in the present investigation is most likely to be mediated by $5-\mathrm{HT}_{2}$ receptors on the vascular smooth muscle cells as demonstrated in porcine ophthalmic and ciliary arteries. ${ }^{2}$ 5-HT can also cause vessels to dilate by acting on $5-\mathrm{HT}_{1}$ receptors on the endothelium to release $\mathrm{NO}^{28}$ but this does not appear to be significant in the bovine anterior ciliary artery as neither removal of the endothelium nor incubation with L-NNA increased its sensitivity to this agonist.

The vasoconstrictor ET-1 is released by damaged or hypoxic endothelium and increased plasma concentrations of ET-1 are associated with several vascular diseases (reviewed by Haynes and $\mathrm{Webb}^{29}$ ) including some types of glaucoma. ${ }^{30}$ ET-1 induces vasoconstriction by stimulation of $\mathrm{ET}_{\mathrm{A}}$ (and to a lesser extent $\mathrm{ET}_{\mathrm{B}}$ receptors) on the vascular smooth muscle but can also induce dilatation by stimulating $\mathrm{ET}_{\mathrm{B}}$ receptors on the endothelial cells. ${ }^{18} \mathrm{ET}_{\mathrm{B}}$ mediated release of endothelium derived vasodilators does not appear to modulate ET-1 induced contraction in isolated bovine anterior ciliary arteries as removal of the endothelium did not increase the vascular sensitivity to this agonist.

In conclusion, the results of the present study show that the endothelium does not modulate responses of isolated bovine anterior ciliary arteries to a variety of constrictor agonists. Furthermore, there does not appear to be a significant basal release of $\mathrm{NO}$ by this artery when studied in an isometric system in vitro. This suggests that endothelial cell dysfunction associated with cardiovascular diseases is unlikely to significantly reduce blood flow to the ciliary body.
This work was supported in part by grants from the Royal College of Surgeons of Edinburgh and the Royal School for the lege of Surgeons of Edin
Blind, Edinburgh, UK.

1 Yao $\mathrm{K}$, Tschudi M, Flammer J, et al. Endotheliumdependent regulation of vascular tone of the porcine dependent regulation of vascular tone of the porcine
ophthalmic artery. Invest Ophthalmol Vis Sci 1991;32:17918 .

2 Haefliger IO, Flammer J, Lüscher TF. Heterogeneity of endothelium-dependent regulation in ophthalmic and ciliary arteries. Invest Ophthalmol Vis Sci 1993;34:1722-30.

3 Meyer P, Flammer J, Lüscher TF. Endothelium-dependent regulation of the ophthalmic microcirculation in the perfused porcine eye: role of nitric oxide and endothelins. Invest Ophthalmol Vis Sci 1993;34:3614-21.

4 Lüscher TF, Vanhoutte PM. Dysfunction of the release of endothelium-dependent relaxing factor. In: Simionescu M, Simionescu M, eds. Endothelial cell dysfunction. New York: Plenum Press, 1991:65-102.

5 Flammer J. To what extent are vascular factors involved in the pathogenesis of glaucoma? In: Kaiser HJ, Flammer J, Hendrickson P, eds. Ocular blood flow: new insights into the pathogenesis of ocular diseases. Basel: Karger, 1995:12-39.

pathogenesis of ocular diseases. Basel: Karger, 1995:12-39.
6 Nathanson JA, McKee M. Identification of an extensive sysNathanson JA, McKee M. Identification of an extensive sys-
tem of nitric oxide-producing cells in the ciliary muscle and tem of nitric oxide-producing cells in the ciliary muscle and outflow pathway of the
Sci $1995 ; 36: 1765-73$.

7 Nathanson JA, McKee M. Alterations of ocular nitric oxide synthase in human glaucoma. Invest Ophthalmol Vis Sci 1995;36: 1774-84.

8 Schuman JS, Erickson K, Nathanson JA. Nitrovasodilator effects on intraocular pressure and outflow facility in monkeys. Exp Eye Res 1994;58:99-105.

9 Mulvany MJ, Halpern W. Mechanical properties of vascular smooth muscle cells in situ. Nature 1976;260:617-19.

10 Mulvany MJ, Halpern W. Contractile properties of small arterial resistance vessels in spontaneously hypertensive and normotensive rats. Circ Res 1977;411:19-26.

11 Nyborg NCB, Korsgaard N, Nielsen PJ. Active wall tension-length curve and morphology of isolated bovine retinal small arteries: important feature for pharmacodynamic studies. Exp Eye Res 1990;51:217-24.

12 Yu D-Y, Alder VA, Cringle SJ. In vitro characterisation of the mechanical properties of canine ophthalmic artery. Exp Eye Res 1990;51:729-34

13 Nyborg NCB, Nielsen PJ. Beta-adrenergic receptors regulating vascular smooth muscle tone are only localised to the intraocular segment of the long posterior ciliary artery in bovine eye. Surv Ophthalmol 1995;39:S66-75.

14 Osol G, Cipolla M, Knutson S. A new method for mechanically denuding the endothelium of small $(50-150 \mu \mathrm{m})$ arteries with a human hair. Blood Vessels 1989;26:320-4.

15 Lüscher TF, Vanhoutte PM. The endothelium: modulator of cardiovascular function. Boca Raton: CRC Press, 1990:1228.

16 Carrier GO, White RE. Enhancement of $\alpha_{1}$ and $\alpha_{2}-$ adrenergic agonist-induced vasoconstriction by removal of endothelium in rat aorta. I Pharmacol Exp Ther 1985;232: 682-7.

17 Buckley CH, Hadoke PWF, O'Brien CJ. The use of isolated ocular arteries in vitro to define the pathology of vascular ocular arteries in vitro to define the pathology of vascular
changes in glaucoma. Br f Ophthalmol 1997;81:599-607.

changes in glaucoma. Br f Ophthalmol 1997;81:599-607.
18 Lüscher TF, Boulanger CM, Yang Z, et al. Interactions between endothelium-derived relaxing and contracting factors in health and cardiovascular disease. Circulation 1993;87(Suppl V):V36-44.

19 Nielsen PJ, Nyborg NCB. Calcium antagonist-induced relaxation of the prostaglandin $\mathrm{F}_{2 \alpha}$ response of isolated calf retinal resistance arteries. Exp Eye Res 1989;48:329-35.

20 Deussen A, Sonntag M, Vogel R. L-Arginine-derived nitric oxide: a major determinant of uveal blood flow. Exp Eye Res 1993;57:129-34

21 Seligsohn EE, Bill A. Effects of $\mathrm{N}^{\mathrm{G}}$-nitro-L-arginine methyl ester on the cardiovascular system of the anaesthetised rabbit and on the cardiovascular response to thyrotropin releasing hormone. Br F Pharmacol 1993;109:1219-25.

22 Yu D-Y, Alder VA, Su E-N, et al. Agonist response of human isolated posterior ciliary artery. Invest Ophthalmol Vis Sci 1992;33:48-54.

23 Ohkubo H, Chiba S. Pharmacological analysis of vasoconstriction of canine ophthalmic and ciliary arteries to alphastriction of canine ophthalmic and ciliary arteries to a

24 Ohkubo H, Chiba S. Responses of isolated canine ophthal mic and ciliary arteries to vasoactive substances. $\mathscr{f} p n \mathcal{F}$ mic and ciliary arteries to

25 Ohkubo H, Chiba S. Vascular reactivities of isolated and perfused human ciliary arteries. Fpn $\mathcal{F}$ Pharmacol 1988;32: $450-6$.

26 Miller VM, Vanhoutte PM. Endothelial $\alpha_{2}$-adrenoceptors in canine pulmonary and systemic blood vessels. Eur F Pharmacol 1985;18:123-9.

27 Vanhoutte PM. Hypercholesterolaemia, atherosclerosis and release of endothelium-derived relaxing factor by aggregating platelets. Eur Heart f 1991;12(Suppl E):25-32.

28 Houston DS, Vanhoutte PM. Comparison of serotonergic receptor subtypes on the smooth muscle and endothelium of the canine coronary artery. F Pharmacol Exp Ther 1988; 244:1-10.

29 Haynes WG, Webb DJ. The endothelin family of peptides. Local hormones with diverse roles in health and disease? Clin Sci 1993;84:485-500.

30 Sugiyama T, Moriya S, Oku H, et al. Association of endothelin-1 with normal tension glaucoma: clinical and fundamental studies. Surv Ophthalmol 1995;39:S49-56. 